\title{
François Moureau (dir.), Marie-Christine Gomez- Géraud, Philippe Antoine, Itinéraires littéraires du voyage
}

\section{Roberta Sapino}

\section{(2) OpenEdition \\ 1 Journals}

\section{Edizione digitale}

URL: http://journals.openedition.org/studifrancesi/1679

DOI: 10.4000/studifrancesi. 1679

ISSN: 2421-5856

\section{Editore}

Rosenberg \& Sellier

\section{Edizione cartacea}

Data di pubblicazione: 1 novembre 2014

Paginazione: 654-656

ISSN: 0039-2944

\section{Notizia bibliografica digitale}

Roberta Sapino, «François Moureau (dir.), Marie-Christine Gomez-Géraud, Philippe Antoine, Itinéraires littéraires du voyage », Studi Francesi [Online], 174 (LVIII | III) | 2014, online dal 01 novembre 2014, consultato il 18 septembre 2020. URL : http://journals.openedition.org/studifrancesi/1679; DOI : https://doi.org/10.4000/studifrancesi. 1679

Questo documento è stato generato automaticamente il 18 settembre 2020.

\section{cc) (†) $\odot$}

Studi Francesi è distribuita con Licenza Creative Commons Attribuzione - Non commerciale - Non opere derivate 4.0 Internazionale. 


\section{François Moureau (dir.), Marie- Christine Gomez-Géraud, Philippe Antoine, Itinéraires littéraires $d u$ voyage}

Roberta Sapino

\section{NOTIZIA}

FRANÇOIS MOUREAU (dir.), MARIE-CHRISTINE GOMEZ-GÉRAUD, PHILIPPE ANTOINE, Itinéraires littéraires du voyage, Travaux de Littérature, XVI, Paris, ADIREL, 2013, pp. 366.

1 Che il récit di viaggio possa essere letto come oggetto essenzialmente letterario è un'idea relativamente recente, ricorda François MOUREAU nella presentazione del volume (pp. 7-8), affermatasi nel corso del xIX secolo contestualmente allo sviluppo del giornalismo e della letteratura industriale. Tuttavia, lo statuto di tale scrittura resta ambiguo: costantemente in bilico tra rigore cronachistico e libertà di invenzione, la letteratura di viaggio rifugge le classificazioni e costituisce uno spazio caleidoscopico che i contributi raccolti da Moureau si propongono di esplorare, osservandone principalmente le declinazioni di area francese dal medioevo al contemporaneo, ma non trascurando qualche incursione in terra straniera.

«La littérature de voyage est-elle de la littérature?» ci si chiede in chiusura della prefazione, e la stessa domanda riecheggia in tutta la prima sezione del volume, intitolata "Voyageurs et voyageuses: une écriture?». Qui Christine FERLAMPIN-ACHER ("Le Voyage d'Outremer" de Bertrandon de la Broquière: récit de pèlerinage, rapport d'espionnage ou récit de voyage?, pp. 11-22) ci guida tra le pagine di un testo del primo Quattrocento in cui un travestimento diventa la chiave per la trasformazione dell'autore in personaggio e dello scripturaire in écriture; Elisabeth SCHNEIKERT (Montaigne et le corps du voyage. «Assaggiamo di parlar un poco questa altra lingua», pp. 23-31) osserva come nel Journal di 
Montaigne l'esperienza del viaggio sia mediata da quella del corpo malato, e come l'italiano, lingua allo stesso tempo familiare e straniera, costituisca per l'autore una maniera di dire la propria condizione attraverso un doppio movimento di distacco e di analisi; Samuel THÉVOZ (Une «étrange nature»: l'exploration du Baltistan ou l'émergence d'un imaginaire féminin dans le "Voyage d'une Parisienne dans l'Himalaya" de Marie de UjfalvyBourdon, pp. 33-47) si sofferma poi su un testo tardo-ottocentesco che, pur inserendosi nel filone piuttosto diffuso dei récits de femmes de savants, emerge in virtù della sua capacità non soltanto di ridiscutere lo status di donna, ma anche di ripensare il genere della scrittura d'esplorazione al femminile all'interno dello spazio letterario. Un approccio di tipo genetico permette a Jessica DESCLAUX (Quand le récit de voyage dialogue avec l'enquête: l'exemple d'“Une enquête aux pays du Levant" de Maurice Barrès, pp. 49-59) di ricostruire una scrittura che si appropria della forma dialogica dell'inchiesta per spingerla ai confini del récit de témoignage, mentre Lidia GONZÁLEZ MENÉNDEZ (Gabrielle Roy journaliste ou le voyage comme découverte, pp. 61-70) conclude mettendo in luce le particolarità dei reportage di Gabrielle Roy, figura fondamentale della letteratura canadese del Novecento, la cui attività giornalistica rimane in gran parte da studiare.

3 La seconda sezione, "Saveurs et images du voyage», si concentra sul viaggio come esperienza sensoriale, e sulle possibilità di rappresentazione che si aprono quando le immagini affiancano il racconto verbale nel tentativo di restituire la fisicità di un luogo. Caroline PRUD'HOMME (Un monde de saveurs. La mise en mots de l'expérience culinaire chez quelques voyageurs du Moyen Âge, pp. 73-79) analizza, in forma e contenuto, le scarse ma significative notazioni riguardanti il cibo presenti nei récits di viaggio medievali, rilevando come queste contribuiscano a indurre nel pubblico un senso di familiarità, ma anche di eccezionalità; Irini APOSTOLOU (Images réelles-images fictives de l'Orient aux $X V I I I^{e}$ et $X_{I X}^{e}$ siècles: le regard des artistes-voyageurs, pp. 81-93) conduce uno studio sull'evoluzione delle rappresentazioni iconografiche dell'Oriente mediterraneo tra Settecento e Ottocento, avendo cura di considerarne gli aspetti tecnici (l'invenzione del dagherrotipo, ad esempio), il valore simbolico (non solo documenti esplicativi, ma garanzie di veridicità del viaggio) e le implicazioni estetiche e ideologiche; Claude REICHLER (Savants en voyages et en images, pp. 95-108) si interroga su come l'immagine assolva alla funzione epistemica, testimoniale ed estetica della scrittura di viaggio, focalizzandosi in particolare su quella a scopo scientifico prodotta tra il diciassettesimo e il diciannovesimo secolo e riguardante la montagna; Danièle mÉAux (Photographes voyageurs en Europe orientale, pp. 109-120), infine, si concentra sul voyage de photographe: opera ibrida di testo e fotografia, individuale o collaborativa, sviluppatasi nel secondo Novecento, la cui indefinitezza generica ben corrisponde alla volontà di raccontare l'eterogeneità di una terra come quella dell'Est Europa postcomunista.

«De la Méditérranée aux Orients» è l'itinerario proposto nella terza sezione del volume, che si apre con tre contributi accomunati dalla riflessione sul viaggio come modalità di percezione del tempo: Richard CRESCENZO («Par six ou sept ans que j’ai demeuré à Rome...». Vigenère voyageur d'Italie, pp. 123-134) mostra come nell'opera di Vigenère le notazioni sui soggiorni italiani testimonino di un'avventura intellettuale tale da indurre l'autore a ripensare la sua relazione con i testi antichi; Anne-Sophie DE FRANCESCHI-GERMAIN (Témoignages d'un monde qui change: les écrits viatiques face aux soubresauts du temps, pp. 135-145) rileva l'influenza dei cambiamenti politici e religiosi del xvi secolo sull'esperienza del viaggiatore nel bacino mediterraneo, ipotizzando che ciò abbia contribuito al passaggio da una concezione circolare del tempo a una lineare; 
Giuseppina TARDANICO (Ruines et mélancolie chez les voyageurs français en Sicile au début du XIX siècle: l'exemple du marquis de Foresta et du comte de Forbin, pp. 147-155) avanza di due secoli per guidarci in Sicilia e osservare come le rovine greche diventino, agli occhi del maquis de Foresta e del comte de Forbin, punto di incontro tra magnificenza e assenza, morte e vita, alterità e prossimità di una gloria antica da ricreare grazie all'immaginazione e alla parola. Carmen DEPASQUALE (Malte des voyageurs au XVIII siècle, pp. 157-168) ricostruisce le diverse percezioni dell'arcipelago maltese - inteso come spazio fisico e come luogo di esperienza - che emergono dai resoconti dei novelli Cavalieri di Malta del diciottesimo secolo; Sarga Moussa (Critique du voyage. L'exemple de la correspondance de Flaubert en Orient, pp. 169-176) mostra come la corrispondenza privata dall'Egitto rappresenti per Flaubert un luogo fondamentale di riflessione sui limiti del linguaggio, la cui capacità di dire il reale è costantemente minata dall'incombere del cliché; Arzu ETENSEL ILDEM (Jean de Thévenot et Evliya Çelebi: le commun et l'étrange, pp. 177-187) compie una dettagliata analisi comparativa delle relazioni del viaggio al Cairo del francese Jean de Thévenot e dell'ottomano Evliya Çelebi per comprendere in che modo la lente soggettiva della cultura d'origine influenzi la descrizione di uno stesso fenomeno. Segue l'intervento di Guy GaLAZKa (Le sacré et le profane en Terre sainte. Au croisement de deux géographies des voyages, pp. 189-198), che osserva come l'enorme incremento del numero di viaggiatori-turisti in Palestina registrato nel corso del XIX secolo abbia comportato una maggior volontà di render conto anche degli aspetti più pratici, emotivi e aneddotici di un'esperienza che non appare più come esclusivamente religiosa; poi Sylviane ALBERTAN-COPPOLA (Des récits de voyage à la collection Prévost: les Chinoises dans tous les états, pp. 199-207) identifica le fonti che sono alla base dell'Histoire générale des voyages e, concentrandosi sui passaggi dedicati alla descrizione delle donne cinesi, conclude rimarcando il ruolo fondamentale degli observateurs en chambre come Prévost nel processo di elaborazione di un sapere enciclopedico; Arlette FRUET (À propos de quelques îles voyageuses, pp. 209-218) ci accompagna alla ricerca delle isole, più o meno immaginarie, che nutrivano la fantasia e la geografia mentale dei navigatori del XVI e XvII secolo.

5 L'ibridità formale che caratterizza la scrittura di viaggio stimola le riflessioni raccolte nella quarta sezione, «Poétiques du voyage». Véronique MAGRI (Reformulation et récit de voyage, pp. 221-230) analizza le modalità in cui la riformulazione - che è insieme ripetizione e rinnovamento - contribuisce a restituire testualmente le problematiche dell'incontro con l'Alterità; Odile GANNIER (Auto-dénigrement et autodérision de l'écrivain voyageur: une rhétorique du chleuasme, pp. 231-242) e Philippe MÉNARD (L'écriture du récit de voyage: les références du narrateur au voyageur dans la version française du "Devisement $d u$ monde" de Marco Polo, pp. 243-253) si concentrano invece sulla presenza autoriale nella scrittura, il primo interrogandosi su motivazioni ed esiti di quelle dichiarazioni di modestia, più o meno esplicite e più o meno sincere, che abbondano nelle parole degli autori e viaggiatori ottocenteschi per poi scomparire pressoché completamente nel secolo successivo, forse in virtù di una parallela democratizzazione delle pratiche del viaggio e della scrittura, il secondo rilevando come nella versione francese del Milione il je di Marco Polo sia costantemente soppiantato da quello del redattore Rustichello da Pisa. A seguire, Jean-Claude LABORIE (Pour une poétique du récit hétérologique: le paradigme du voyage au Brésil au XvI siècle, pp. 255-263) ribadisce la necessità di considerare la scrittura di viaggio non valutandone la pertinenza al genere scientifico o romanzesco, ma riconoscendone il collocarsi in una tensione dialettica tra i due generi come cifra 
della presenza dell'Altro nella materialità del testo; Catherine BRouÉ e Marc-André MARCHAND (La figure du voyageur Cavelier de La Salle au service de l'image d'auteur, pp. 265-277) valutano i complessi rapporti tra il viaggiatore-scrittore in carne e ossa e le diverse forme dell'ethos discorsivo così come emergono nelle lettere dalla Louisiana di Cavelier de La Salle e nel Journal historique di Joutel. Il contributo di Sylvie REQUEMORAGROS (Le genre «mitoyen» en question: le cas de l'épisode algérien de Regnard, pp. 279-288) ritorna sulla questione della letteratura di viaggio intesa come genre mitoyen tra Storia $\mathrm{e}$ romanzo, analizzando principalmente le procedure di détournement générique in atto nella Provençale di Regnart e nella Relation de l'esclavage des sieurs de Fercourt et Regnart pris sur mer par les corsaires d'Alger (1678-1679), mentre Isabelle BES HOGTHON (De la relation éclairée au récit d'inspiration réaliste: l'évolution diachronique du récit de voyage au XIX siècle. Une étude de cas, pp. 289-299) ripercorre l'evoluzione di tale scrittura nel contesto della storia letteraria ottocentesca, dalla pseudo-oggettività dell'Illuminismo alla soggettività romantica alla pseudo-oggettività realista, mettendone in luce le diverse tematiche e modalità discorsive.

L'ultima sezione, più breve, raccoglie tre interventi dedicati al ventesimo secolo: dalla vastissima opera di Morand, Dominique LANNI (Du voyageur écrivain à l'écrivain voyageur: Paul Morand, pp. 303-312) seleziona un corpus composto dalle opere degli anni Venti per comprendere il processo di costituzione della figura autoriale, e identifica nel giro del mondo del 1925 - e negli scritti che ne sono conseguiti - un momento fondante per il passaggio dal voyageur écrivain all'écrivain voyageur; Frank LESTRINGANT (Gide voyage: du "Voyage au Congo aux Retouches à mon "Retour de l'U.R.S.S."» (1925-1937), pp. 313-336) si sofferma su due grandi viaggi di Gide, pubblicati a una decina d'anni di distanza l'uno dall'altro, analizzandone particolarità tematiche, aspetti formali e questioni di ricezione per mostrare come la volontà di viaggiare per vedere "derrière les décors" si concretizzi in una scrittura sempre più distante dalle tentazioni di esotismo e sempre più prossima a una testimonianza intrisa di protesta politica; Guillaume THOUROUDE ( «Lieux de mémoire» et «non-lieux» dans l'oeuvre viatique de Jean Rolin, pp. 337-346) chiude osservando come l'opera di Jean Rolin apporti un contributo importante alla letteratura di viaggio contemporanea, configurandosi come un'esplorazione del paese d'origine, dei suoi paesaggi, della sua evoluzione e delle sue contraddizioni, e restituendo l'immagine caleidoscopica di uno spazio articolato tra luoghi e memoria, territorio e identità.

7 In calce al volume, un utile indice dei nomi citati, una sintetica presentazione degli autori dei contributi e la rubrica bibliografica con le pubblicazioni recenti dei membri dell'ADIREL. 